Eur. J. Clin. Chem. Clin. Biochem.

Vol. 32, 1994, pp. 377-381

(c) 1994 Walter de Gruyter \& Co. Berlin - New York

\title{
Plasma Pancreatic Lipase Activity: From Analytical Specificity to Clinical Efficiency for the Diagnosis of Acute Pancreatitis
}

\author{
By J. M. Lessinger and G. Férard \\ Laboratoire de Biochimie Appliquée, UFR des Sciences Pharmaceutiques, Université Louis Pasteur de Strasbourg. \\ Illkirch, France
}

(Received September 23, 1993/February 8, 1994)

Summary: Using five procedures (turbidimetry with the Boehringer Mannheim kit and with a home made reagent, reflectometry with the Eastman Kodak kit, colorimetry with the Sigma kit, and UV spectrophotometry with the Wako kit), lipase activity was assayed in the same group of 60 healthy adults and in 30 patients suffering from acute pancreatitis ( $n=197$ samples) as well as in a purified and stabilized preparation of human pancreatic lipase. Results indicated considerable inter-assay discrepancies for the mean values of the patients' results: catalytic activity concentrations differed by a factor of up to 16 according to the measurement procedures. For each method, mean patients' results were also expressed as multiples of the upper limit of normal values. This method of presentation did not significantly improve the intra-assay agreement, with maximal relative differences as high as 13 -fold. When each method was calibrated with the same material (human pancreatic lipase), the inter-assay agreement was considerably improved. The causes of inter-assay disagreement are discussed in detail, and the necessity for a validated lipase calibrator is stressed, in order to improve the efficiency of the information transmitted by clinical laboratories to clinicians. A strategy is proposed, which includes development of a reference method and reference material, and a study of inter-assay commutability of secondary calibrators for a set of methods.

\section{Introduction}

Pancreatic lipase (E. C. 3.1.1.3, triacylglycerol acylhydrolase) is often considered to be a more sensitive and specific marker than $\alpha$-amylase and pancreatic isoamylase for the diagnosis of acute pancreatitis $(1-4)$. However, discrepancies in the reported data are frequent and may have at least three causes. The selection of patient populations, particularly with regard to the prevalence of acute pancreatitis and the retained criteria for the diagnosis of acute pancreatitis differ from one study to another (5-6). Furthermore, the chosen decision limit for lipase activity varies considerably between studies $(3,6,7)$. Finally, routine methods used for the measurement of serum lipase activity involve turbidimetric, reflectometric, and visible or UV spectrophotometric techniques, differing from one study to another.
In this study, our goal was to:

- compare the reaction conditions of the methods of measurement commonly used in clinical laboratories and to see if they fulfil requirements for the specific determination of pancreatic lipase activity;

- compare the results of plasma lipase activity in patients suffering from acute pancreatitis with regard to the analytical procedures;

- evaluate the advantage of a proper lipase calibrator for the improvement of the interassay agreement of results transmitted to the clinicians.

\section{Materials and Methods}

Table 1 shows the five methods used in this study. All the assays utilize detergents (most often bile salts) at supramicellar concen- 


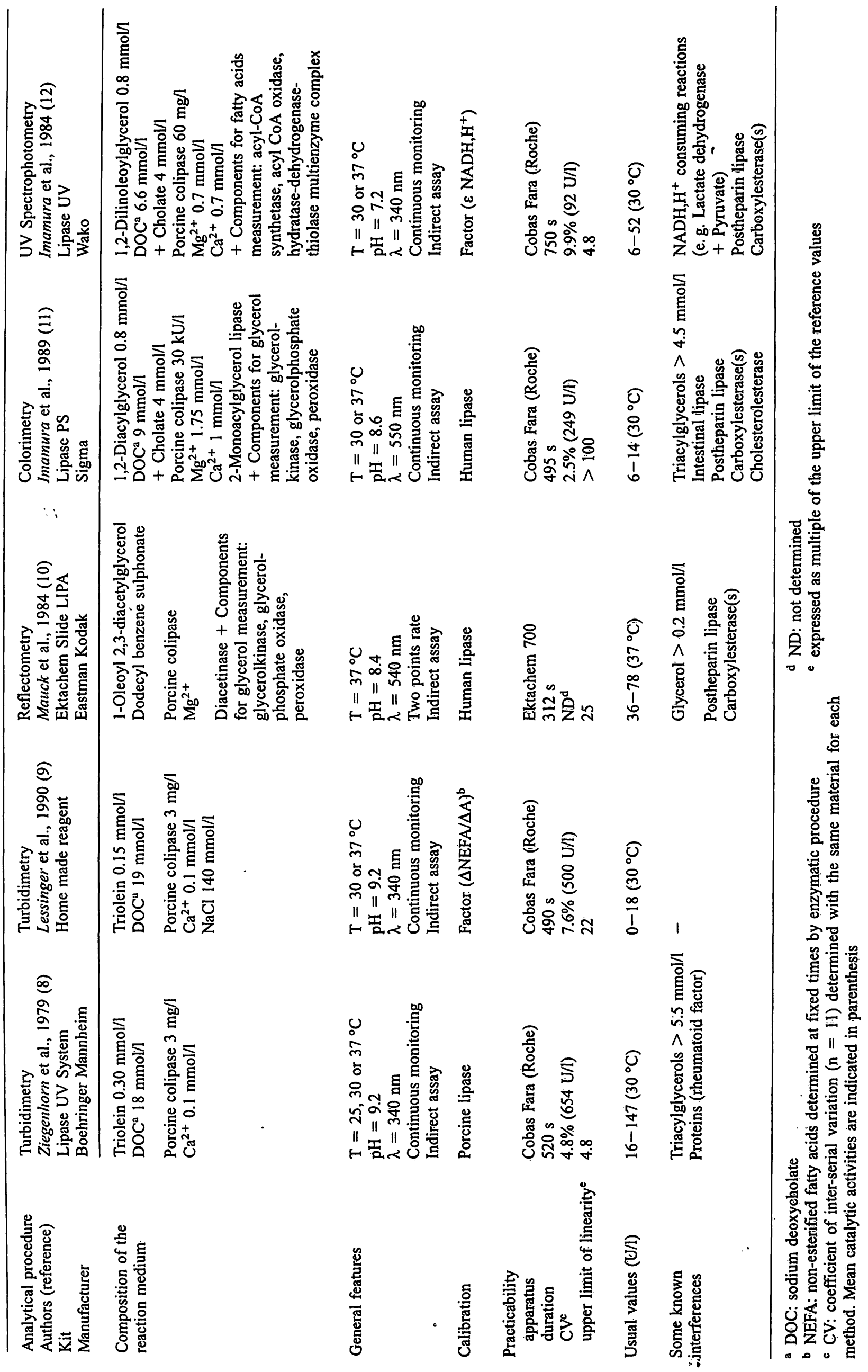


trations, thereby reducing the lag phase and inhibiting all the lipolytic activities. The deoxycholate concentration varies between 6.6 and $19 \mathrm{mmol} / \mathrm{l}$. The Eastman Kodak reagent contains dodecyl benzene sulphonate instead of a bile salt. Colipase, added in excess, specifically reverses this inhibition and induces a considerable activation of the enzyme. One or two divalent cations $\left(\mathrm{Ca}^{2+}, \mathrm{Mg}^{2+}\right)$ are always added in these modern methods. The $\mathrm{pH}$ of the reaction media varies from 7.2 to 9.2. All the procedures can be automated and the reaction can be continuously monitored, except for the reflectometric test. Most of the lipase assays are indirect in the sense that they do not determine the number of ester bonds hydrolysed per time unit (13), unlike the titrimetric procedures derived from the Cherry \& Crandall method (14). For this reason, indirect methods are often calibrated with the help of an enzyme standard prepared from human or porcine pancreas. In the present study, a purified stable human pancreatic lipase was also used for interassay comparison (15).

Blood samples from 30 patients suffering from acute pancreatitis were collected on lithium heparinate, and 197 plasma samples were assayed for lipase activity by five methods on the same apparatus (Cobas Fara, Roche) at $30^{\circ} \mathrm{C}$, except for the reflectometric technique which was performed at $37^{\circ} \mathrm{C}$ on an Ektachem 700. Plasma samples were also obtained from 60 apparently healthy adults (30 men and 30 women) aged 20-60 years, and the reference values of plasma lipase activity were established for each method. For the four commercialized reagents, manufacturer's recommendations were followed without modification.

The mean and standard deviations (SD) of the five series of results were calculated for healthy and patient groups. For healthy adults, intervals corresponding to mean $\pm 2 \mathrm{SD}$ were considered as the reference ranges of lipase activity concentrations in plasma.

\section{Results}

In our hands, all the procedures were easy to perform, could be adapted to emergency situations, and were reproducible. Nevertheless, the UV spectrophotometric assay (Wako) was less precise in terms of interserial reproducibility. Furthermore, ranges of linearity vary considerably from 5 times the upper limit of reference values (Boehringer Mannheim) to more than 100 times (Sigma).

Figure 1a shows a typical example of patterns observed for a patient suffering from acute pancreatitis with a favourable evolution. Values attained a peak during the observation period, but the actual values varied greatly depending on the methods. When the activity concentrations were divided by the upper limit of reference values (fig. 1b), values still displayed considerable differences, depending on the method of measurement. When the mean values of lipase activity concentrations of all patients' samples were considered (tab. 2), we noted that they differed by a factor of up to 16 according to the method used, but the results were strongly correlated $(0.95<r<0.98)$. For each of the 30 patients studied, the maximum of lipase activity was observed at the same time for each method of measurement. At this time, the activity concentrations were always in the same order: Wako $<$ Sigma $<$ home made reagent $<$ Boehringer $<$ Kodak. When patients' results were ex-
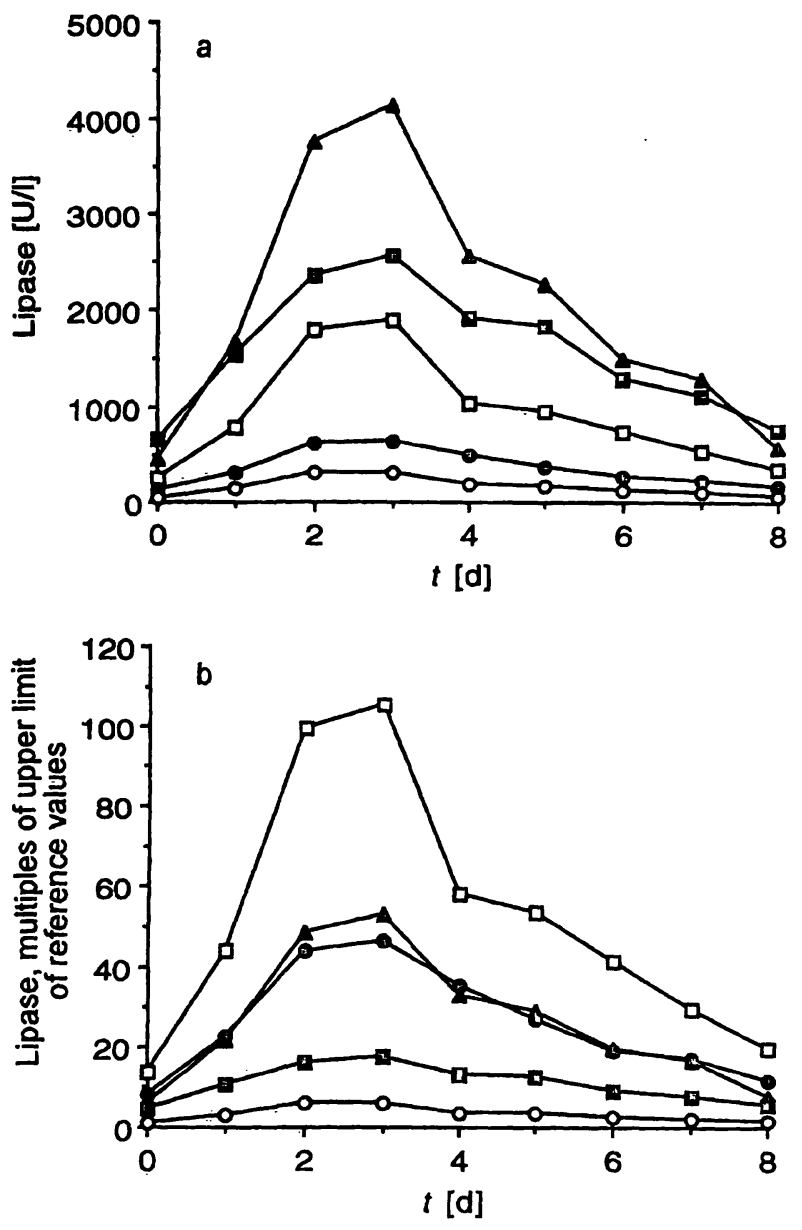

Fig. 1 Plasma of a patient diagnosed as having acute pancreatitis was assayed for lipase activity over several days, using five different procedures ( $\Delta$ Eastman Kodak reagent; i Boehringer Mannheim reagent; $\square$ Home made reagent; 0 Sigma reagent; $O$ Wako reagent, see Materials and Methods). Results are expressed in U/I (fig. la) and in multiples of the upper limit of reference values (fig. 1b).

Tab. 2 Comparison of three modes of expression of lipase activity.

For each method, the results are expressed in catalytic activity concentrations (mean value \pm standard deviation), in multiples of the upper limit of reference values and the activity relative to that determined in a purified preparation of pancreatic lipase.

\begin{tabular}{llll}
\hline Reagent & Lipase & & \\
\cline { 2 - 4 } & $\begin{array}{l}\text { Catalytic } \\
\text { activity } \\
\text { concentration } \\
\text { [U/l] }\end{array}$ & $\begin{array}{l}\text { Multiples of } \\
\text { the upper } \\
\text { limit of } \\
\text { reference } \\
\text { values }\end{array}$ & $\begin{array}{l}\text { Activity } \\
\text { relative to } \\
\text { purified } \\
\text { enzyme }\end{array}$ \\
\hline $\begin{array}{l}\text { Boehringer } \\
\text { Mannheim }\end{array}$ & $765 \pm 602$ & 5.2 & 1.17 \\
$\begin{array}{c}\text { Home made } \\
\text { reagent }\end{array}$ & $420 \pm 412$ & 23.3 & 0.84 \\
$\begin{array}{c}\text { Eastman } \\
\text { Kodak }\end{array}$ & $1516 \pm 1089$ & 19.4 & 0.93 \\
$\begin{array}{l}\text { Sigma } \\
\text { Wako }\end{array}$ & $294 \pm 202$ & 21.0 & 1.18 \\
\hline & $92 \pm 82$ & 1.8 & 1.00 \\
\hline
\end{tabular}


pressed as multiples of the upper limit of reference values, they still differed (by as much as 13-fold), but they were in a different order (Wako $<$ Boehringer $<$ Kodak $<$ Sigma $<$ home made reagent). By using a purified and stabilized preparation of human pancreatic lipase (15) as a standard, it was possible to compare the results obtained with the five methods. As shown in table 2, when the reaction rate observed with the purified preparation was assigned a value of 1.0 for each method, the inter-assay agreement was considerably improved; the lipase activity by different methods then varied by a factor of 1 to 1.4. Each method was not calibrated with the commercial standards for the different procedures, because inter-method discrepancies were found to be higher for these materials than for patient samples (not shown).

\section{Discussion}

Several routine methods are suitable for the assay of lipase activity in emergency situations in terms of precision, rapidity and practicability. Nevertheless, considerable inter-assay discrepancies were observed in the degree of increase in the plasma lipase activity. They were not due to inaccuracies in the temperature or volume settings, since the same apparatus was used for performing four of the five tested methods, and four procedures employed a calibrator. Similarly, differences in the selection of healthy subjects cannot explain interassay disagreement, since samples from the same apparently healthy subjects were retained to establish reference ranges for the five methods.

There are several possible reasons for these discrepancies, involving the calibration procedures and the analytical specificity of the methods. The enzyme calibrators should exhibit the same catalytic properties as the enzyme determined in human samples; this is not always the case (9), especially with regard to the requirements for effectors, and thermodependance. As most of the tested methods use different calibrators, inter-method discrepancy may be due to differences in the origin of the calibrator, and to effects of different procedures for the purification and stabilization of the calibrator enzyme, as well as the chosen matrix. All these variables might affect the catalytic properties of calibrators, and lead to the observed inter-assay discrepancies. Moreover, the calibrators proposed by the manufacturers are not titrated against the same reference method. The reference procedures also differ with respect to several variables such as temperature, substrate concentration, mode of emulsification of the substrate, type and concentration of the added bile salt, and supplementation with colipase $(16,17)$.
This study also shows that strong correlations between different assays do not necessarily mean that the considered assays exhibit identical analytical specificities. This is clearly illustrated by the great discrepancies observed when patients' results are expressed as multiples of the upper limit of the reference ranges. In fact, the validity of this approach depends on an identical specificity of the compared methods with regard to the plasma lipolytic activities from healthy subjects and patients. Obviously, this requirement was not fulfilled for all the tested methods of measurement. Several authors have pointed out that various enzymes may interfere in some assays $(6,18,19)$. Differences in the nature of the substrate (1,2-diacylglycerol, asymmetric or homogeneous triacylglycerol), the size of micelles and the type of detergent may affect analytical specificity. Furthermore, different forms of pancreatic lipase, at least two, have been observed in plasma samples of patients suffering from acute pancreatitis $(20,21)$. The profile of pancreatic lipase isoforms, which is not the same in healthy subjects and in cases of acute pancreatitis, may be a source of inter-assay disagreement $(20,21)$. We also have to keep in mind that different cut-off values were used when comparative studies were performed to evaluate the efficiency of lipase for the diagnosis of acute pancreatitis. These cut-offs varied from one to nine when lipase activities were expressed in multiples of the upper limit of the reference ranges $(5,6)$.

\section{Conclusion}

Lipase provides an interesting illustration of some general features of clinical enzymology. As for all catalytic activities, results are method dependent, but differences are much more marked for lipase than for other enzymes when results are expressed in catalytic concentrations (U/l). At present, the consensus for the use of an excess of a detergent and of colipase is not sufficient to ensure inter-assay agreement. One pecularity of lipase assays in clinical enzymology is the use of calibrators in most of the kits. If a calibration procedure is used to reduce the effect of drift on the results, it can also be a source of inaccuracy, if its catalytic properties are not the same as those of human enzyme. Moreover, there is not yet a reference method for the determination of lipase activity.

Based on the results of the present study, we propose here a strategy to improve the efficiency. of information transmitted to clinicans. The first step is the development of a reference method and of a lipase reference material exhibiting the same catalytic properties as the plasma "pancreatitis" lipase. The second step is the certification of the reference material by the reference method. Then, routine methods havie to be tested against the 
reference method, by assaying a series of both patients' samples and the reference material in order to verify commutability of the latter product. Commutability refers to the ability of an enzyme material to show interassay activity changes comparable to those of the same enzyme in human plasma (22). If commutability of the reference material with patients' samples is observed for a given set of methods, reference and routine methods will show similar accuracy, and there will be inter-assay agreement with the reference method. Moreover, the reference material can be used for the selection of secondary calibrators by verifying that the inter-method ratio of activity is the same for secondary materials as for the reference preparation. Furthermore, in such a set of methods, the reference material, titrated against the reference method, could be employed to assign values to secondary calibrators. Obviously, other sets of methods excluding the reference method may exhibit agreement, but accuracy would not be ensured. In this case, it seems likely that two different sets of methods would not show the same clinical sensitivity and specificity for the diagnosis of acute pancreatitis. Nevertheless, such a strategy employing a "reference system" (both reference method and certified reference material) in a defined set of methods could probably be applied to other markers in clinical chemistry, in order to improve the efficiency of the information transmitted by the clinical laboratory to clinicians.

\section{Acknowledgements}

We wish to thank $E$. Laurent for her technical assistance and the Community Bureau of Reference (BCR) of the European Communities for the financial support of the preparation of the purified and stabilized material of human pancreatic lipase.

\section{References}

1. Lott, J. A., Patel, S. T., Sawhney, A. K., Kazmierczak, S. C. \& Love, J. E. Jr (1986) Assays of serum lipase: Analytical and clinical considerations. Clin. Chem. 32, 1290-1302.

2. Tietz, N. W. (1988) Amylase measurement in serum. Old myths die hard. J. Clin. Chem. Clin. Biochem. 26, 251-253.

3. Clavien, P. A., Burgan, S. \& Moossa, A. R. (1989) Serum enzymes and other laboratory tests in acute pancreatitis. Br. J. Surg. 76, 1234-1243.

4. Van Lente, F. \& Kazmierczak, S. C. (1989) Immunologicallyderived pancreatic amylase, pancreatic lipase, and total amylase compared as predictor of pancreatic inflammation. Clin. Chem. 35, 1542.

5. Lott, J. A., Ellison, E. C. \& Applegate, D. (1989) The importance of objective data in the diagnosis of pancreatitis. Clin. Chim. Acta 183, 33-40.

6. Tietz, N. W. \& Shuey, D. F. (1993) Lipase in serum - the elusive enzyme: An overview. Clin. Chem. 39, 746-756.

7. Panteghini, M., Pagini, F. \& Bonora, R. (1993) Clinical and analytical evaluation of a continuous enzymatic method for measuring pancreatic lipase activity. Clin. Chem. 39, 304308.

8. Ziegenhorn, J., Neumann, U., Knitsch, K. W. \& Zwez, W. (1979) Determination of serum lipase. Clin. Chem. 25, 1067.

9. Lessinger, J. M., Férard, G., Balaskä, C. \& Arzoglou, P. (1990) Lipase turbidimetric and nephelometric asssays: Further studies on calibration and clinical interest. Clin. Chem. Enzymol. Comms. 3, 167-181.

10. Mauck, J. C., Weaver, M. S. \& Stanton, C. (1984) Development of a Kodak Ektachem clinical chemistry slide for serum lipase. Clin. Chem. 30, 1058-1059.

11. Imamura, S. \& Misaki, H. (1989) An enzymatic method using 1,2-diglyceride for pancreatic lipase test in serum. Clin. Chem. 35,1126

12. Imamura, S. \& Misaki, H. (1984) A sensitive method for assay of lipase activity by coupling with $\beta$-oxydation enzymes of fatty acid. In: Selected Topics in Clinical Enzymology (Goldberg, D. M. \& Werner, M., eds) pp. 73-77, Walter de Gruyter, Berlin-New York, vol. 12 .

13. Férard, G. \& Lessinger, J. M. (1992) L'activité de la lipase pancréatique humaine: Revue des méthodes et recommendations générales. Ann. Biol. Clin. 50, 133-141.

14. Cherry, I. S. \& Crandall, L. (1932) The specificity of pancreatic lipase: Its appearance in the blood after pancreatic injury. Amer. J. Physiol. 100, 266-273.

15. Lessinger, J. M., Tavridou, A., Arzoglou, P. L. \& Férard, G (1992) Interest of using a purified, stable and commutable preparation of human pancreatic lipase in indirect assays. Anal. Lett. 25, 1453-1568.

16. Rick, W. (1969) Kinetischer Test zur Bestimmung der Serumlipaseaktivität. Z. Klin. Chem. Klin. Biochem. 7, 530-539.

17. Tietz, N. W. \& Repique, E. V. (1973) Proposed standard method for measuring lipase activity in serum by a continuous sampling technique. Clin. Chem. 19, 1268-1275.

18. Demanet, C., Goedhuys, W., Haentjens, M., Huyghens, L., Blaton, V. \& Gorus, F. (1992) Two automated fully enzymatic assays for lipase activity in serum compared: Positive interference from post-heparin lipase activity. Clin. Chem. 38, 288-292.

19. Tetrault, G. A. (1991) Lipase activity in serum measured with Ektachem is often increased in nonpancreatic disorders. Clin. Chem. 37, 447-451.

20. Lessinger, J. M., Arzoglou, P. L. \& Férard, G. (1986) Evidence for multiple forms of pancreatic lipase in human plasma. Adv. Clin. Enzymol. 3, 139-150.

21. Lott, J. A. \& Lu, C. L. (1991) Lipase isoforms and amylase isoenzymes: Assays and application in the diagnosis of acute pancreatitis. Clin. Chem. 37, 361-368.

23. Fasce, C. F., Rej, R., Copeland, W. H. \& Vanderlinde, R. E. (1973) A discussion of enzyme reference materials: Applications and specifications. Clin. Chem. 19, 5-9.

Professor G. Férard

Laboratoire de Biochimie

Appliquée

UER des Sciences Pharmaceutiques

Université Louis Pasteur

F-67400 Illkirch

France 
